\title{
Model Predictive Static Programming Guidance Method with Trajectory Way-points Constraints
}

\author{
Zhe Luo, Xinsan Li*, Lixin Wang \\ Xi'an High-Tech Institution, Xi'an 710025, Shaanxi, China.
}

How to cite this paper: Zhe Luo, Xinsan Li, Lixin Wang. (2021) Model Predictive Static Programming Guidance Method with Trajectory Way-points Constraints. Journal of Applied Mathematics and Computation, 5(4), 349-353.

DOI: 10.26855/jamc.2021.12.013

Received: November 17, 2021

Accepted: December 10, 2021

Published: December 23, 2021

*Corresponding author: Xinsan Li, Xi'an High-Tech Institution, Xi'an 710025, Shaanxi, China.

Email: xinsan_2006@163.com

\begin{abstract}
Model predictive static programming (MPSP) guidance method is proposed for tactical ballistic missile penetration in near space. The proposed MPSP guidance method satisfies both way-points as well as final outputs constraints. The trajectory shape of the tactical ballistic missile is adjusted in near space by setting a series of way-points constraints. Effectiveness of the MPSP guidance method is verified with minimum dynamic pressure constraints (MDPC). Simulation results show that the MPSP guidance method can well meet the way-points constraints and the final outputs constraints in near space. By controlling the trajectories way-points of the tactical ballistic missile in near space, the shape of the trajectory is adjusted, which can improve the maneuver penetration capability for tactical ballistic missiles.
\end{abstract}

\section{Keywords}

Guidance, MPSP, Way-Points Constraint, Dynamic Pressure Constraint

\section{Introduction}

There is a renewed interest around the world in prediction-correction guidance law [1-3]. Pseudo-spectral method is a prediction-correction method with obvious advantages in computational efficiency [4]. But it requires careful selection of the basis function and configuration poles. MPC method is another predictive-correction guidance method with potential for online applications [5, 6]. It predicts the outputs in a limited time, and the controls are solved iteratively based on the deviations between the predicted values and the expected values. Padhi and Oza have recently proposed an MPSP guidance method with final multi-constraints [7]. It combines MPC with approximate dynamic programming (ADP) method and the optimal control problem is solved by minimizing the control energy with final constraints. Traditional optimal guidance method transforms the optimal control problem into a two-point boundary value problem. The MPSP guidance method only relies on the static covariate variables with the same outputs dimension when updating the controls. In addition, the MPSP guidance method solves the sensitivity matrix recursively, and the calculation efficiency is very high. Unlike the transcription method such as pseudo spectral method, it does not need to optimize parameters with numerical calculation. It is suitable for solving very complex guidance problems, such as ballistic guidance problems [9, 10].

The main contents of this paper include: the basic principle of the MPSP guidance method and the validity verification of MPSP guidance method with minimum dynamic pressure constraints.

\section{MPSP Guidance Method}

For a general form of nonlinear systems, the discrete equations of the states and the outputs are as follows

$$
\begin{aligned}
& \boldsymbol{X}_{k+1}=\boldsymbol{F}_{k}\left(\boldsymbol{X}_{k}, \boldsymbol{U}_{k}\right) \\
& \boldsymbol{Y}_{k}=\boldsymbol{h}\left(\boldsymbol{X}_{k}\right)
\end{aligned}
$$


where $\boldsymbol{X} \in \boldsymbol{R}^{n}, \quad \boldsymbol{U} \in \boldsymbol{R}^{m}, \quad \boldsymbol{Y} \in \boldsymbol{R}^{p}, \quad k=1,2, \ldots, N$. The objective of the MPSP guidance method is to find appropriate controls $\boldsymbol{U}_{k}, \quad k=1,2, \ldots, N-1$, so that the output at the final time step $\boldsymbol{Y}_{N}$ goes to a desired value $\boldsymbol{Y}_{N}^{*}$, and satisfies $\boldsymbol{Y}_{N} \rightarrow \boldsymbol{Y}_{N}^{*}$. The final time output deviation is $\Delta \boldsymbol{Y}_{N}=\boldsymbol{Y}_{N}-\boldsymbol{Y}_{N}^{*}$. Expand $\boldsymbol{Y}_{N}$ Taylor series with respect to $\boldsymbol{Y}_{N}^{*}$ as follows:

$$
\boldsymbol{Y}_{N}=\boldsymbol{Y}_{N}^{*}+\left[\frac{\partial \boldsymbol{Y}_{N}}{\partial \boldsymbol{X}_{N}}\right] d \boldsymbol{X}_{N}+\text { HOT }
$$

where HOT are higher-order terms. Equation (3) ignores the influence of the high-order terms, and the output deviation at the final time is defined as

$$
\Delta \boldsymbol{Y}_{N} \cong d \boldsymbol{Y}_{N}=\left[\frac{\partial \boldsymbol{Y}_{N}}{\partial \boldsymbol{X}_{N}}\right] d \boldsymbol{X}_{N}
$$

Equation (1) and equation (2) are discretized on each node and the following equation is obtained as follows

$$
d \boldsymbol{Y}_{N}=\boldsymbol{A} d \boldsymbol{X}_{1}+\boldsymbol{B} d \boldsymbol{U}_{1}+\ldots+\boldsymbol{B}_{N-1} d \boldsymbol{U}_{N-1}
$$

where

$$
\boldsymbol{A}=\left[\frac{\partial \boldsymbol{Y}_{N}}{\partial \boldsymbol{X}_{N}}\right]\left[\frac{\partial \boldsymbol{F}_{N-1}}{\partial \boldsymbol{X}_{N-1}}\right] \ldots\left[\frac{\partial \boldsymbol{F}_{1}}{\partial \boldsymbol{X}_{1}}\right]
$$

The sensitivity matrix $\boldsymbol{B}_{k}$ between the final output and the control $\boldsymbol{U}_{k}$ is defined as

$$
\boldsymbol{B}_{k}=\left[\frac{\partial \boldsymbol{Y}_{N}}{\partial \boldsymbol{X}_{N}}\right]\left[\frac{\partial \boldsymbol{F}_{N-1}}{\partial \boldsymbol{X}_{N-1}}\right] \ldots\left[\frac{\partial \boldsymbol{F}_{k+1}}{\partial \boldsymbol{X}_{k+1}}\right]\left[\frac{\partial \boldsymbol{F}_{k}}{\partial \boldsymbol{U}_{k}}\right]
$$

$k=1, \ldots, N-1$

The sensitivity matrix $\boldsymbol{B}_{k}$ can be computed recursively. $\boldsymbol{B}_{N-1}^{0}$ is defined firstly, as

$$
\boldsymbol{B}_{N-1}^{0}=\left[\frac{\partial \boldsymbol{Y}_{N}}{\partial \boldsymbol{X}_{N}}\right]
$$

$\boldsymbol{B}_{k}^{0}$ is computed secondly, $k=(N-2),(N-3), \ldots, 1$, as

$$
\boldsymbol{B}_{k}^{0}=\boldsymbol{B}_{k+1}^{0}\left[\frac{\partial \boldsymbol{F}_{k+1}}{\partial \boldsymbol{X}_{k+1}}\right]
$$

$\boldsymbol{B}_{k}, \quad k=(N-1),(N-2), \ldots, 1$, is computed finally as follows

$$
\boldsymbol{B}_{k}=\boldsymbol{B}_{k}^{0}\left[\frac{\partial \boldsymbol{F}_{k}}{\partial \boldsymbol{U}_{k}}\right]
$$

$\boldsymbol{B}_{k}^{0}$ is computed recursively by the equation(7)-(10), thus greatly reducing the calculation load. From (3), we have $(N-1) m$ unknowns, and $p$ equations. Usually, $p<(N-1) m$. This system has multiple sets of solutions and the following performance objective is taken for solving the equation (3), as

$$
J=\frac{1}{2} \sum_{k=1}^{N-1}\left(\boldsymbol{U}_{k}^{0}-d \boldsymbol{U}_{k}\right)^{T} \boldsymbol{R}_{k}\left(\boldsymbol{U}_{k}^{0}-d \boldsymbol{U}_{k}\right)
$$

where $\boldsymbol{U}_{k}^{0}, k=1, \ldots, N-1$, is the previous controls and $d \boldsymbol{U}_{k}$ is the corresponding controls deviation. $\boldsymbol{R}_{k}$ is the weighting matrix. The deviation of the controls corresponding to the $k$ th step can be obtained by the optimal control theory.

$$
d \boldsymbol{U}_{k}=-\boldsymbol{R}_{k}^{-1} \boldsymbol{B}_{k}^{T} \boldsymbol{A}_{\lambda}^{-1}\left(d \boldsymbol{Y}_{N}-\boldsymbol{b}_{\lambda}\right)+\boldsymbol{U}_{k}^{0}
$$


Thus, the controls in step $k$ is as follows

$$
\boldsymbol{U}_{k}=\boldsymbol{U}_{k}^{0}-d \boldsymbol{U}_{k}=\boldsymbol{R}_{k}^{-1} \boldsymbol{B}_{k}^{T} \boldsymbol{A}_{\lambda}^{-1}\left(d \boldsymbol{Y}_{N}-\boldsymbol{b}_{\lambda}\right)
$$

where

$$
\begin{aligned}
& \boldsymbol{A}_{\lambda}=\left[-\sum_{k=1}^{N-1} \boldsymbol{B}_{k} \boldsymbol{R}_{k}^{-1} \boldsymbol{B}_{k}^{T}\right] \\
& \boldsymbol{b}_{\lambda}=\left[\sum_{k=1}^{N-1} \boldsymbol{B}_{k} \boldsymbol{U}_{k}^{0}\right]
\end{aligned}
$$

When using MPSP guidance method, it needs to make an initial guess on the controls, and then update the current controls by iterative calculation, until the final condition is met, i.e. $\boldsymbol{Y}_{N} \rightarrow \boldsymbol{Y}_{N}^{*}$.

\section{Results}

\subsection{Missile Parameters}

If the tactical ballistic missile is typically aerodynamically controlled and hence the missile must meet the minimum dynamic pressure constraint required for aerodynamically controlling. It is not allowed to fly into the thin atmosphere. If the missile's range is as far as possible, the highest point of the flight trajectory should be as high as possible. The dynamic pressure value is constrained at the way-points for low-trajectory penetration in near space. The minimum dynamic pressure condition for pneumatic maneuvering is satisfied.

The outputs at each way-point are selected as follows

$$
\boldsymbol{Y}_{N_{i}}^{i}=\left[\gamma_{m}\left(N_{i}\right) \psi_{m}\left(N_{i}\right) Q_{m}\left(N_{i}\right)\right]^{T}
$$

where $Q_{m}\left(N_{i}\right)$ is the constrained dynamic pressure at each way-point. The MDPC trajectory is designed by constraining the flight path angle, the deflection angle and the dynamic pressure at each way-point. Table 1 is the MDPC way-points constraints, where $N_{1}, N_{2}$ and $N_{3}$ are different way-points.

Table 1. MDPC way-points constraints

\begin{tabular}{cccc}
\hline Parameters & Value & Unit \\
\hline Way-point, $N_{1}\left(\gamma_{m c}, \psi_{m c}, Q_{c}\right)$ & $(0.0,0.0,120000.0)$ & $\left(\operatorname{deg}, \operatorname{deg}, \mathrm{N} / \mathrm{m}^{2}\right)$ \\
Way-point, $N_{2}\left(\gamma_{m c}, \psi_{m c}, Q_{c}\right)$ & $(0.0,0.0,120000.0)$ & $\left(\mathrm{deg}, \mathrm{deg}, \mathrm{N} / \mathrm{m}^{2}\right)$ \\
Way-point, $N_{3}\left(\gamma_{m c}, \psi_{m c}, z_{c}\right)$ & $(0.0,0.0,12000.0)$ & $(\mathrm{deg}, \mathrm{deg}, \mathrm{m})$ \\
\hline
\end{tabular}

The iteration number of MPSP is 4 . Figure 1 shows that the dynamic pressure constraints are met well at way-points $N_{1} \quad(\mathrm{t}=40.3 \mathrm{~s})$ and $N_{2} \quad(\mathrm{t}=100.7 \mathrm{~s})$. The dynamic pressure deviation is in the range of $1.0 \mathrm{~N} / \mathrm{m} 2$. Figures 2,3 , 4, and 5 show the comparison of $a_{z}, V_{m}, \gamma_{m}, z_{m}$ with MDPC and WCC3. The range of the states and the control for MDPC are larger compared with WCC1. The $a_{z}$ is smooth between each way-point. The deviation of the flight path angle is within the range of 0.1 degree at each way-point. The $z_{m}$ deviation can be controlled within $1.0 \mathrm{~m}$ at each way-point. Since the MDPC constrains the dynamic pressure at the trajectory way-points $N_{1} \quad(\mathrm{t}=40.3 \mathrm{~s})$ and $N_{2} \quad(\mathrm{t}=100.7 \mathrm{~s})$, the flight height is greatly increased compared to the WCC3. The tactical ballistic missile can meet the minimum dynamic pressure condition for pneumatic maneuvering through the dynamic pressure constraints at the way-points. The shape of the trajectory can be adjusted, thereby enhancing the complexity of the trajectory in near space, and greatly improving the penetration capability of the ballistic missile.

\section{Conclusions}

This paper extends the MPSP guidance method and obtains the guidance method for tactical ballistic missile satisfying both way-points as well as final outputs constraints. The trajectory shape of the tactical ballistic missile is adjusted in near space by setting a series of way-points constraints on the trajectory. The main advantages of the proposed MDPC 
maneuvering strategy are: the minimum dynamic pressure constraints required for pneumatic control, and the propulsion system works more stable under approximately constant dynamic pressure, and autonomous penetration of hypersonic vehicles can be achieved by adjusting the dynamic pressure at the waypoint in near space.

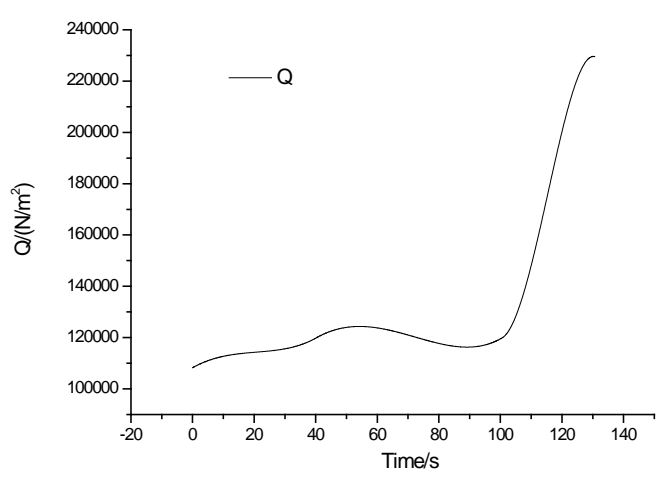

Figure 1. Dynamic pressure $Q$ histories.

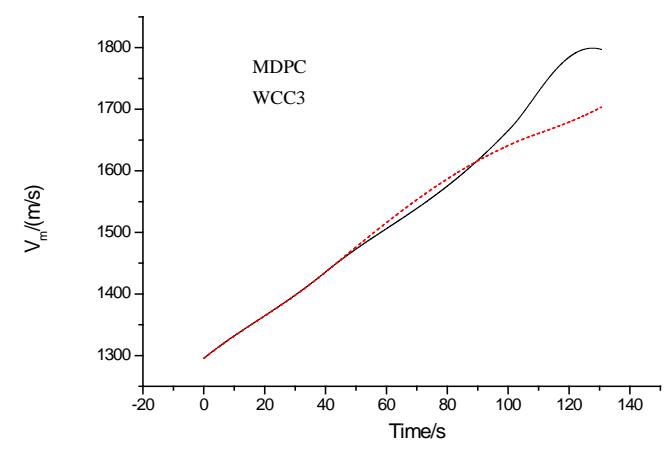

Figure 3. Velocity $V_{m}$ histories.

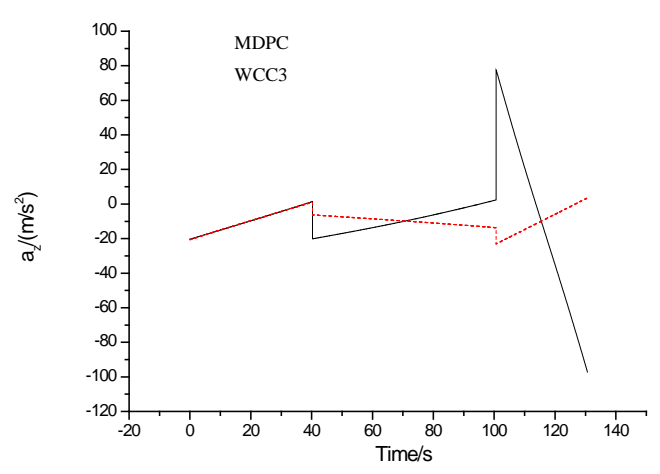

Figure 2. Normal acceleration $a_{z}$ histories.

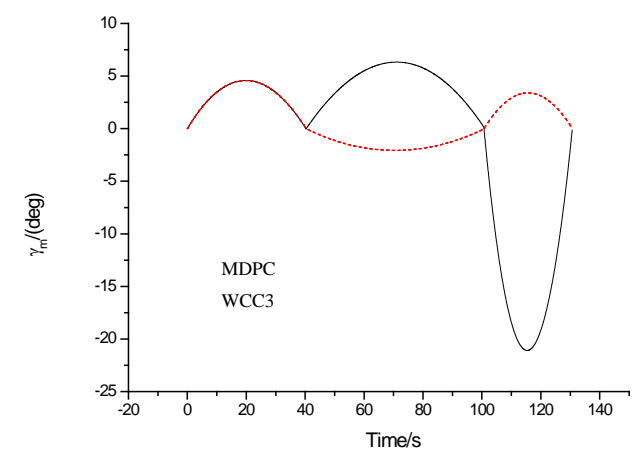

Figure 4. Flight path angle $\gamma$ histories.

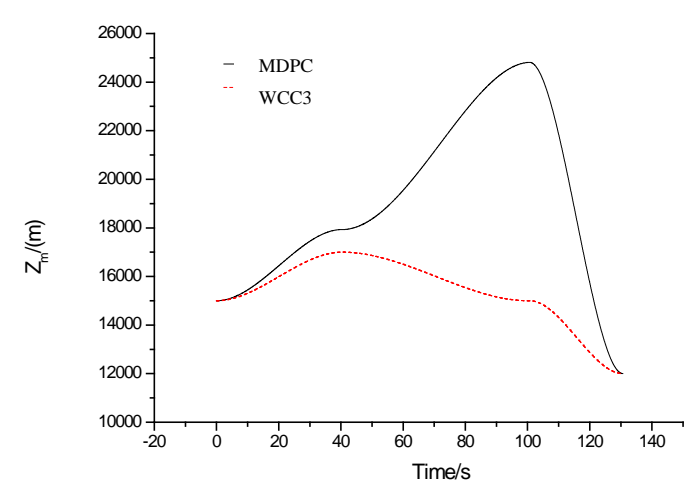

Figure 5. Displacement $Z_{m}$ histories.

\section{Funding}

This research was funded by NATIONAL NATURAL SCIENCE FOUNDATION OF CHINA, grant number 61503390 . 


\section{References}

[1] Maity, A., Oza, H. B., and Padhi, R. (2014). Generalized Model Predictive Static Programming and angle-constrained guidance of air-to-ground missiles. Journal of Guidance, Control and Dynamics, 2014, Vol. 39, No. 6, pp. 1-17.

[2] Maity, A., Oza, H. B., and Padhi, R. (2013). Generalized model predictive static programming and its application to 3D impact angle constrained guidance of Air-to-Surface missiles. 2013 American Control Conference (ACC), Washington, DC, USA, 2013, pp. 4999-5014.

[3] Li, X. S., Wang, L. X. (2017). Two-stage Control Guidance of Missile Impact Angle and Flight Time. Journal of National University of Defense Technology, 2017, Vol. 39, No. 6, pp. 6-11.

[4] Gong, Q., Kang, W., and Ross, I. M. (2006). A Pseudospectral Method for The Optimal Control of Constrained Feedback Linearizable Systems. IEEE Transactions on Automatic Control, 2006, Vol. 51, No. 7, pp. 1115-1129.

[5] Cannon, M. (2004). Efficient nonlinear model predictive control algorithms. Annual Reviews in Control, 2004 , Vol. 28, No. 2, pp. 229-237.

[6] Siegers, N., Costello, M. (2005). Model predictive control of a parafoil and payload system. Journal of Guidance, Control and Dynamics, 2005, Vol. 28, No. 4, pp. 816-821.

[7] Padhi, R., Kothari, M. (2009). Model predictive static programming: a computationally efficient technique for suboptimal control design. International Journal of Innovative Computing, Information and Control, 2009, Vol. 5, No. 2, pp. 399-411.

[8] Dwivedi, P. N., Bhattacharya, A., and Padhi, R. (2011). Suboptimal midcourse guidance of interceptors for high speed targets with alignment angle constraint. Journal of Guidance, Control and Dynamics, 2011, Vol. 34, No. 3, pp. 860-877.

[9] Oza, H. B., Padhi, R. (2012). Impact Angle Constrained suboptimal model predictive static programming guidance of air-to-ground missiles. Journal of Guidance, Control and Dynamics, 2012, Vol. 35, No. 1, pp. 153-164. 